\title{
Excellent AUC for joint fluid cytology in the detection/exclusion of hip and knee prosthetic joint infection
}

\author{
Jiri Galloa, Jarmila Juranovab ${ }^{b}$ Michal Svoboda ${ }^{a}$, Jana Zapletalovac
}

\begin{abstract}
Background and Aim. The aim of this study was to evaluate the characteristics of synovial fluid (SF) white cell count (SWCC) and neutrophil/lymphocyte percentage in the diagnosis of prosthetic joint infection (PJI) for particular threshold values.

Methods and Results. This was a prospective study of 391 patients in whom SF specimens were collected before total joint replacement revisions. SF was aspirated before joint capsule incision. The PJl diagnosis was based only on non-SF data. Receiver operating characteristic plots were constructed for the SWCC and differential counts of leukocytes in aspirated fluid. Logistic binomic regression was used to distinguish infected and non-infected cases in the combined data. PJI was diagnosed in 78 patients, and aseptic revision in 313 patients. The areas (AUC) under the curve for the SWCC, the neutrophil and lymphocyte percentages were $0.974,0.962$, and 0.951 , respectively. The optimal cut-off for PJI was 3,450 cells/ $\mu \mathrm{L}, 74.6 \%$ neutrophils, and $14.6 \%$ lymphocytes. Positive likelihood ratios for the SWCC, neutrophil and lymphocyte percentages were 19.0, 10.4, and 9.5, respectively. Negative likelihood ratios for the SWCC, neutrophil and lymphocyte percentages were $0.06,0.076$, and 0.092 , respectively.

Conclusions. Based on AUC, the present study identified cut-off values for the SWCC and differential leukocyte count for the diagnosis of PJI. The likelihood ratio for positive/negative SWCCs can significantly change the pre-test probability of PJI.
\end{abstract}

Key words: prosthetic joint infection, synovial white cell count, neutrophil percentage, lymphocyte percentage, ROC curves

Received: January 4, 2017; Accepted: April 20, 2017; Available online: May 15, 2017 https://doi.org/10.5507/bp.2017.021

${ }^{a}$ Department of Orthopaedics, University Hospital Olomouc and Faculty of Medicine and Dentistry, Palacky University Olomouc, Czech Republic

${ }^{b}$ Department of Hemato-Oncology, University Hospital Olomouc and Faculty of Medicine and Dentistry, Palackyý University Olomouc, Czech Republic

'Department of Medical Biophysics, University Hospital Olomouc and Faculty of Medicine and Dentistry, Palacky University Olomouc, Czech Republic

Corresponding author: Jiri Gallo, e-mail:jiri.gallo@volny.cz

\section{BACKGROUND}

Prosthetic joint infection (PJI) is a feared complication of total joint arthroplasty. Accurate diagnosis of this complication is a key step for rational therapeutic management. To establish a standardized PJI diagnosis procedure, the Musculoskeletal Infection Society (MSIS) introduced a definition based on the presence of strong and standard diagnostic criteria ${ }^{1}$. In addition, several strategies have been proposed to increase the probability of accurate PJI diagnosis depending on individual clinical presentation ${ }^{2}$.

The recommendation is to puncture artificial joints preoperatively when the probability of infection is increased by the erythrocyte sedimentation rate (ESR) and/or raised C-reactive protein (CRP). Some studies have discredited the value of elevated ESR and CRP at least in some patients ${ }^{3}$, which raised the question of the appropriateness of routine preoperative aspiration in all patients indicated for revision surgery regardless of the pretest probability of PJI. Joint fluid samples (JFSs) are sent for microbial, biochemical, and cytological examination (i.e. the synovial white cell count (SWCC) and differential count of leukocytes in synovial fluid). Several studies reported the potential of the SWCC for diagnosing PJI. As a result, national and international guidelines strongly recommend that patients' SWCC and neutrophil percentage be assessed for PJI (ref. $\left.{ }^{4-6}\right)$. However, the proposed cut-off values for the SWCC (ranging from 1,100 to 50,000 cells $/ \mu \mathrm{L}$ ) and differential leukocyte count (in the case the neutrophil count it is between $60 \%$ and $89 \%$ ) remain widely ranging ${ }^{7}$.

The aim of this study was to show the diagnostic characteristics of the SWCC and neutrophil/lymphocyte percentage in the diagnosis of PJI for particular threshold values. Included were all patients who were treated for PJI at our institution and for whom the SWCC and differential leukocyte counts were available.

\section{MATERIALS AND METHODS}

\section{Patient selection}

Overall, 107 joints (105 patients) with PJI were diagnosed and treated at the Department of Orthopaedics, 
University Hospital Olomouc, Czech Republic, between January 2009 and August 2015. A routine set of examinations based on the PJI criteria described here was performed in all patients. However, joint fluid samples were neither obtained nor analyzed in 29 of them $(27 \%)$. Therefore, 78 infected joints (hip or knee) were included in the study (Table 1$)$. The control group $(n=313)$ included patients with total joint arthroplasties (total hip and

Table 1. Basic characteristics of the group of patients revised due to PJI.

\begin{tabular}{|c|c|c|c|}
\hline \multirow{2}{*}{$\begin{array}{l}\text { Variables } \\
\text { Males/Females }\end{array}$} & & \multicolumn{2}{|c|}{ Report } \\
\hline & & $38 / 40$ & $(49 \%$, resp. $51 \%)$ \\
\hline THRs/TKRs & & $27 / 51$ & $(14.4 \% \text {, resp. } 25.1 \%)^{*}$ \\
\hline \multirow[t]{7}{*}{ PJI modes } & Early postoperative infection & 19 & $24.4 \%$ \\
\hline & Delayed infection & 16 & $20.5 \%$ \\
\hline & Haematogenous infection & 27 & $34.6 \%$ \\
\hline & Positive intraoperative culture & 1 & $1.3 \%$ \\
\hline & Relapsing infection & 2 & $2.6 \%$ \\
\hline & Reinfection & 4 & $5.1 \%$ \\
\hline & Direct spreading from surroundings & 9 & $11.5 \%$ \\
\hline SWCC (cells $/ \mu \mathrm{L})$ & & 39,000 & $(28,800-56,800)$ \\
\hline Neutrophil percentage & & $90 \%$ & $(88 \%-92 \%)$ \\
\hline Lymphocyte percentage & & $3 \%$ & $(2.2 \%-4.0 \%)$ \\
\hline Preoperative CRP (mg/L) & & 112 & $(60-176)$ \\
\hline Preoperative ESR (mm/h) & & 59.5 & $(55.0-61.5)$ \\
\hline \multirow[t]{3}{*}{ Preoperative IL-6 (ng/L) } & for both the THR and TKR groups & 63 & $(48-124)$ \\
\hline & for THRs separately & 63 & $(29-132)$ \\
\hline & for TKRs separately & 64 & $(31-173)$ \\
\hline \multirow[t]{3}{*}{ Microbiological microscopy } & Negative & 7 & $9.2 \%$ \\
\hline & Neutrophils & 47 & $61.8 \%$ \\
\hline & Neutrophils and bacteria & 22 & $28.9 \%$ \\
\hline $\begin{array}{l}\text { Age at the time of primary } \\
\text { implantation (years) }\end{array}$ & & 67.6 & $(66.7-72.2)$ \\
\hline Age at the time of revision ( $y$ & & 72.7 & $(69.2-75.9)$ \\
\hline Implant survival (months) & & 21.2 & $(7.1-28.3)$ \\
\hline
\end{tabular}

$\mathrm{THR}=$ total hip replacement TKR = total knee replacement; $\mathrm{PJI}=$ prosthetic joint infection; $\mathrm{SWCC}=$ synovial white cell count; $\mathrm{CRP}=\mathrm{C}$-reactive protein; ESR = erythrocyte sedimentation rate; IL-6 = Interleukin 6; CI = confidence interval.

Data in the table are presented as median and 95\% CI for the median.

* This percentage refers to the proportion of PJI among all the revised THRs, resp. TKRs.

Table 2. List of variables describing the control groups.

\begin{tabular}{llcc}
\hline Variables & & \multicolumn{2}{c}{ Report } \\
\hline Males/Females & In both the aseptic groups & $125 / 188$ & $40.0 \%$, resp. 60.0\% \\
THRs/TKRs & & $161 / 152$ & $(600-780)$ \\
SWCC (cells/ $\mu \mathrm{L})$ & & 700 & $(39 \%-46 \%)$ \\
Neutrophil percentage & & $42 \%$ & $(45 \%-54 \%)$ \\
Lymphocyte percentage & & $50 \%$ & $(2.7-4.0)$ \\
Preoperative CRP (mg/L) & & 3.2 & $(14.2-20.0)$ \\
Preoperative ESR (mm/h) & for both the THR and TKR groups & 17.0 & $(3.6-6.2)$ \\
Preoperative IL-6 (ng/L) & for THRs separately & 4.8 & $(2.2-12.9)$ \\
& for TKRs separately & 4.5 & $(3.6-8.9)$ \\
& Negative & 5.2 & $97.0 \%$ \\
Microbiological microscopy & Neutrophils & 258 & $2.3 \%$ \\
& Neutrophils and bacteria & 6 & $0.7 \%$ \\
Age at the time of primary implantation (years) & & 1 & $(57.3-61.5)$ \\
Age at the time of revision (years) & & 59.4 & $(67.1-70.7)$ \\
Implant survival (months) & & 68.8 & $(117.2-131.2)$ \\
\hline
\end{tabular}

$\mathrm{THR}=$ total hip replacement; TKR = total knee replacement; $\mathrm{SWCC}=$ synovial white cell count $\mathrm{CRP}=\mathrm{C}$-reactive protein; $\mathrm{ESR}=$ erythrocyte sedimentation rate; IL-6 = Interleukin 6 ; $\mathrm{CI}=$ confidence interval.

Data in the table are presented as median and $95 \% \mathrm{CI}$ for the median. 


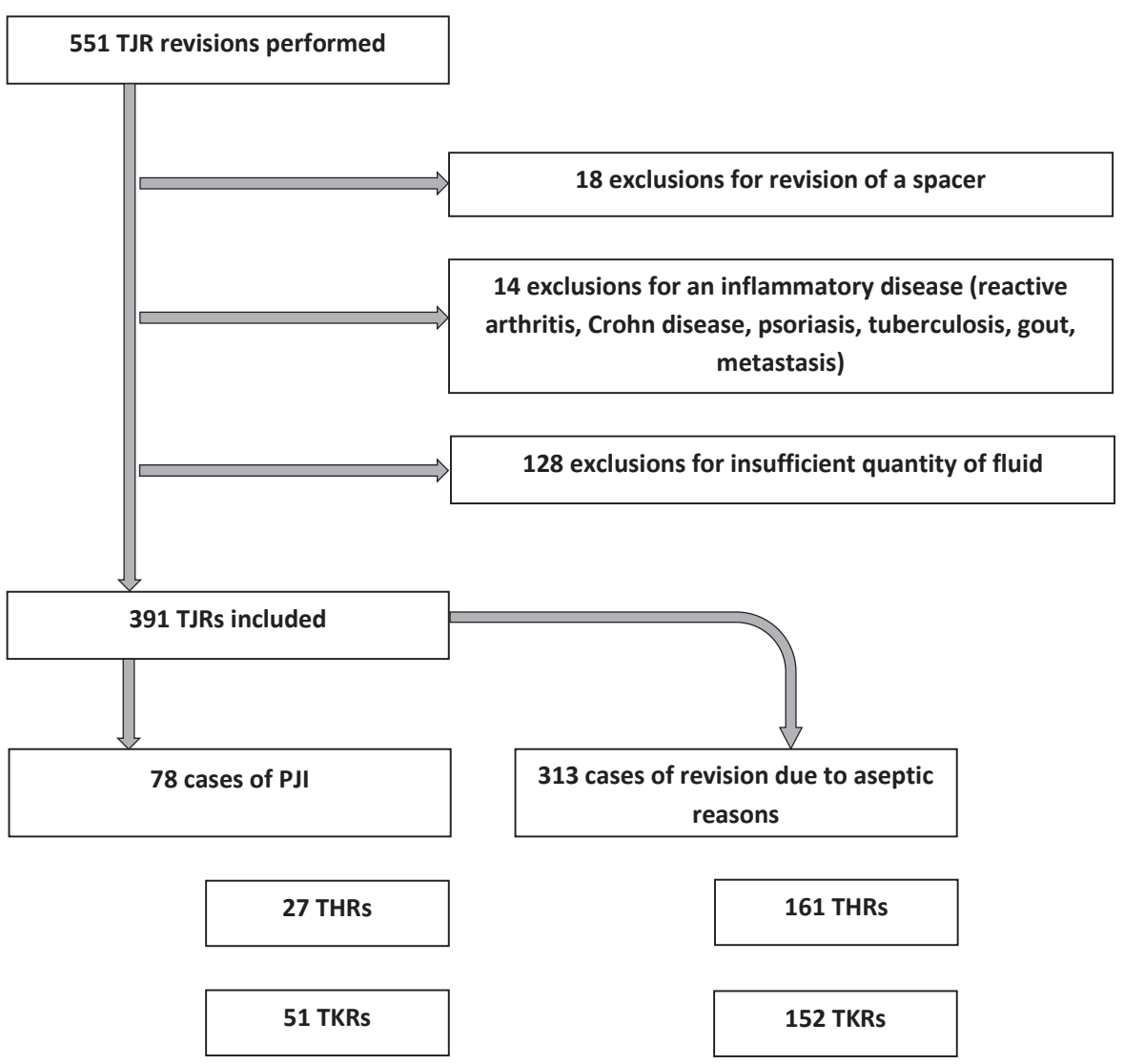

Fig. 1. Flowchart showing the number of included and excluded patients as well as their distribution in the infected and non-infected groups.

$\mathrm{TJR}=$ total joint replacement; $\mathrm{THR}=$ total hip replacement; $\mathrm{TKR}=$ total knee replacement.

knee replacements; THRs and TKRs) revised for aseptic reasons during the same period (Fig. 1). These patients were examined according to the same protocol as the PJI patients (Table 2). As a result, the proportion of PJIs revised for was $19.9 \%(78 / 391)$.

All the revisions were carried out under standard conditions with written informed patient consent, and the study was approved by the local Ethics Committee. The data collection was planned before the index test as well as reference standard. In order to reflect true clinical situations, we included all patients who completed sampling in the study. 37 patients $(47 \%)$ had received antibiotic pretreatment for a median of 25 days (minimum, 1 day) prior to JFS aspiration.

\section{Definition of PJI}

PJI was diagnosed according to the following criteria: 1) presence of a sinus tract communicating with a joint and/or intra-articular pus; 2) coincidentally positive results of histological examination ( 5 or more neutrophils per high power field) and culture of intraoperative samples; 3 ) if only intraoperative culture or histological results were positive, then at least two of the following signs had to be present: high clinical suspicion of infection (acute onset, fever, erythema, edema, persistent local pain, early prosthetic failure, wound healing disturbances, etc.), erythrocyte sedimentation rate $>30 \mathrm{~mm} / \mathrm{h}$, or C-reactive protein elevated more than 1.5 times above the laboratory reference value ${ }^{8}$. Interleukin 6 (IL-6) in the serum was tested in the most recent patients, and results above the laboratory reference value were regarded as positive. Plain radiography was not included in the diagnostic algorithm because of its low specificity with regard to PJI. Aseptic revisions were those that did not fulfill the conditions for PJI.

Early postoperative (onset before the $4^{\text {th }}$ postoperative month), delayed (onset between the $4^{\text {th }}$ to $24^{\text {th }}$ months postoperatively), and haematogenous infections were distinguished ${ }^{9}$. Relapsing infection was defined as a repeated failure of a joint previously revised due to repeated infection by the same bacteria regardless of the time since surgery. Reinfection was defined as PJI caused by bacteria different from the ones cultivated from the same prosthetic joint during the previous revision. "Positive intraoperative culture" meant positive intraoperative culture in patients revised for reasons that were assumed to be aseptic. Direct spread from infected surroundings was assumed, such as when erysipelas was followed by PJI of knee arthroplasty (Table 1).

\section{Synovial white cell count and differential count of leukocytes in synovial fluid}

In order to minimize the risk of false positive results, synovial joint fluid aspiration was performed prior to surgical joint capsule incision. A minimum of $2 \mathrm{~mL}$ per method under study was collected, and then 
transported in special transport tubes with EDTA (Ethylenediaminetetraacetic acid) preventing agglutination of JFSs. The SWCC was performed using Sysmex XE-5000 (Sysmex Corporation; Kobe, Japan) automated hematology analyzer in the body fluid mode according to the instructions of the manufacturers. The resulting values are given in number of cells $/ \mu \mathrm{L}$.

The differential count of leukocytes (i.e. neutrophil/ lymphocyte percentage) was determined manually by microscope: smears of joint fluid samples were performed that were stained by Pappenheim's panoptic staining method, and then evaluated under microscope per 100 cells (leukocytes) at 1000-times magnification. The resulting values are given as ratio or percentage.

\section{Culture}

Joint fluid aspiration was sampled for culture in the same manner as for the SWCC and differential count of leukocytes. After opening the joint, samples of granulation tissue were taken from at least three different sites (one from the capsule and two from host bone beds). Further, hard swabs were taken from the implant surfaces. Retrieved implants were sent for sonication in specific transport boxes. After sampling for microbial examination, systemic or local antibiotics were administered to all patients with the exception of 37 who had received antibiotic treatment before the surgery. Overall, we collected at least 5 samples (range, 5-9) per patient. All materials were transported to the laboratory immediately after sampling. Specimens were processed by the laboratory within 2 hours after collection according to the standardized laboratory protocols for aerobic and anaerobic cultivation in PJI ( ref. $\left.^{8}\right)$. Sonication was performed according to the protocol published elsewhere ${ }^{10}$.

Joint fluid aspirations were considered positive if there were any signs of growth, including in enrichment broth, but concordance between these and intraoperative findings was required. Intraoperative samples were considered positive for infection if the same bacterium was cultured from at least two different operative sites ${ }^{11}$.

\section{Tissue sampling}

Experienced pathologists collaborating with us on other implant-related studies performed the histology. The conclusion was positive when a mean of $\geq 5$ polymorphonuclear leukocytes (PMNs) were seen on at least ten high power fields (HPFs) at 400-times magnification ${ }^{12}$.

\section{Study design, statistical analysis}

During the study neither the microbiologists nor the cytologists knew the clinical and histological data. The data were collected and continuously entered into electronic spreadsheets (Microsoft Excel, Microsoft Corp., WA, USA). After the completion of the study, one of us who had not participated neither in the diagnosis nor therapy of patients, classified the patients in the study into two groups (those with and those without PJI) according to the criteria described above. Neither the SWCC nor neutrophil/lymphocyte percentage were part of the diagnostic verification.
All the statistical calculations including sensitivity, specificity, accuracy, positive and negative predictive values (PPVs, NPVs), positive and negative likelihood ratios $(\mathrm{LR}+,-)$, the diagnostic odds ratio (DOR) and their confidence intervals were performed using IBM SPSS Statistics v. 22. (USA). Other parameters were evaluated according to type using appropriate tests and the same statistical package. Statistical significance was set at $P=0.05$.

To dichotomize continuous data, it is necessary to determine an optimal cut-off value. Values above or equal to this value are then considered as positive. Values below this point were negative. We used the receiver operating characteristic (ROC) curves plotting the true positive rate (sensitivity) against the false positive rate (1-specificity). The area under the curve (AUC) served as a single measure characterizing the discriminative ability of a test across the full range of cut-offs. An optimal cut-off value was determined using the Youden index $(\mathrm{J})$, $\left(\right.$ ref. $\left.^{13}\right)$.

In order to be able to interpret all the data available from our JFS analysis (i.e. SWCC, neutrophil and lymphocyte percentages) simultaneously, the binomic logistic regression model was used. The first model involved the distinct outcome; the second model worked with binary outcomes $(0=$ negative outcome in relation to a particular cut-off point; 1 = positive outcome of the method in relation to a particular cut-off point). Prob value in this logistic binomic model is resulting probability that a particular patient has an infected (if prob $>0.5$ ) or aseptic (if prob < 0.5 ) joint with sensitivity $88.9 \%$, specificity $99.2 \%$, the false positive rate $0.8 \%$, and the false negative rate $11.1 \%$.

\section{RESULTS}

\section{Culture}

The most frequent pathogen was Staphylococcus aureus $(24.4 \%)$ followed by coagulase-negative staphylococci (17.9\%), beta-haemolytic streptococci (14.1\%) and viridans streptococci (6.4\%). Polymicrobial infections were classified in $5.1 \%$ of cases. Culture negative infections were found in $29.5 \%$ of cases.

\section{Synovial white cell count and differential count of leukocytes in synovial fluid}

We compared the SWCC, neutrophil and lymphocyte percentages between patients with PJI and aseptic failure. SWCC medians were as follows: 39,000 cells $/ \mu \mathrm{L}$ vs. 700 cells $/ \mu \mathrm{L}$ (for THR and TKR); 36,300 cells/ $\mu \mathrm{L}$ vs. 610 cells/ $\mu \mathrm{L}$ (for THR), and 39,600 cells/ $\mu \mathrm{L}$ vs. 740 cells/ $\mu \mathrm{L}$ (for TKR). Medians for neutrophil percentage were 90\% vs. $42 \%$ (for THR and TKR); $91 \%$ vs. $46 \%$ (for THR), and $89 \%$ vs. $38 \%$ (for TKR). Medians for lymphocyte percentage were $3 \%$ vs. $50 \%$ (for THR and TKR); $3 \%$ vs. $47 \%$ (for THR), and 3\% vs. 54\% (for TKR). The MannWhitney U test assessed the probability less than 0.0001 that the infected and aseptic groups are identical as far as the SWCC, neutrophil and lymphocyte percentages are concerned. 

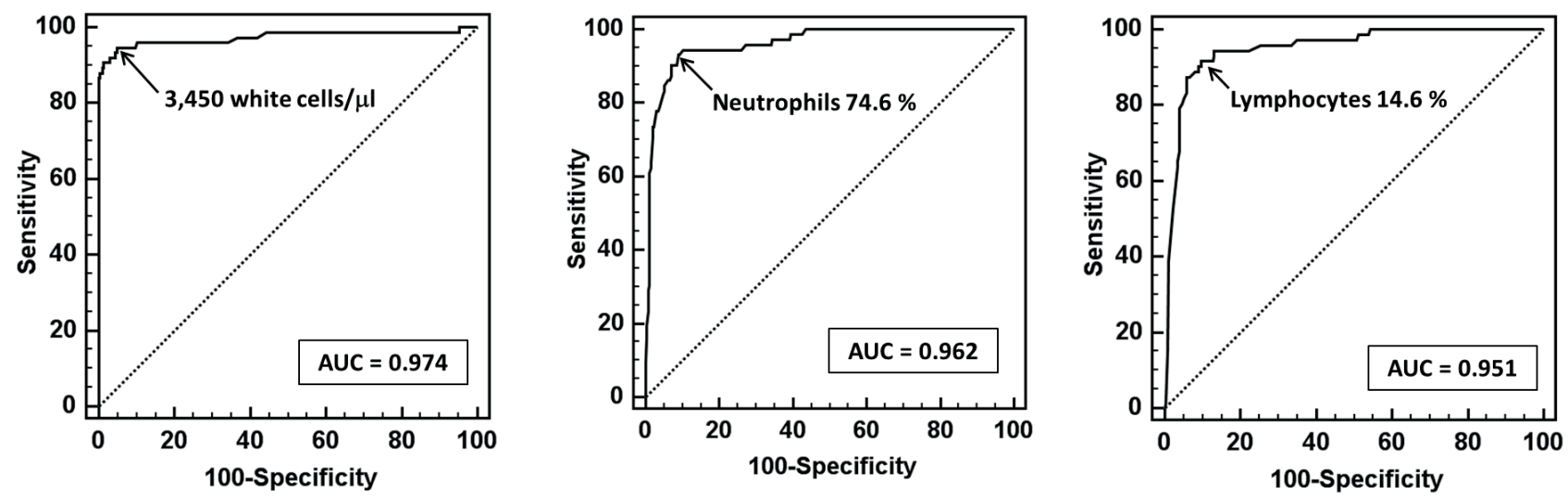

Fig. 2. Receiver operating characteristic (ROC) curves for a) the synovial white cell count; b) neutrophil percentage; and c) lymphocyte percentage. The diagonal dashed line is the line of non-discrimination. The larger the area under the curve, the higher the sensitivity and specificity, and thus the power of the diagnostic test. AUC = area under the curve.

Table 3. Diagnostic characteristics for the SWCC, percentage of neutrophils and percentage of lymphocytes for all the groups and for THRs or TKRs separately.

\begin{tabular}{|c|c|c|c|c|c|c|c|c|c|}
\hline & \multicolumn{3}{|c|}{ SWCC } & \multicolumn{3}{|c|}{ Neutrophil percentage } & \multicolumn{3}{|c|}{ Lymphocyte percentage } \\
\hline & $\begin{array}{l}\text { THR + } \\
\text { TKR }\end{array}$ & THR & TKR & $\begin{array}{l}\text { THR + } \\
\text { TKR }\end{array}$ & THR & TKR & $\begin{array}{l}\text { THR + } \\
\text { TKR }\end{array}$ & THR & TKR \\
\hline Cut-off* & 3,450 & 4,100 & 3,200 & $74.60 \%$ & $76.50 \%$ & $72.80 \%$ & $14.60 \%$ & $14.60 \%$ & $19.90 \%$ \\
\hline Sensitivity & 94.7 & 92.0 & 96.0 & 93.1 & 95.7 & 93.9 & 91.7 & 95.7 & 93.9 \\
\hline Specificity & 95.0 & 96.8 & 95.9 & 91.1 & 93.8 & 90.0 & 90.3 & 93.0 & 84.6 \\
\hline PPV & 82.6 & 82.1 & 88.9 & 74.4 & 73.3 & 78.0 & 72.5 & 71.0 & 69.7 \\
\hline NPV & 98.6 & 98.7 & 98.6 & 97.9 & 99.2 & 97.5 & 97.5 & 99.2 & 97.3 \\
\hline LR+ & 19.0 & 28.3 & 23.5 & 10.4 & 15.3 & 9.4 & 9.5 & 13.6 & 6.1 \\
\hline LR- & 0.06 & 0.08 & 0.04 & 0.076 & 0.046 & 0.068 & 0.092 & 0.047 & 0.072 \\
\hline DOR & 339 & 341 & 560 & 136.9 & 332.6 & 138.2 & 102.5 & 289.4 & 84.7 \\
\hline
\end{tabular}

SWCC = synovial white cell count; THR = total hip replacement; TKR = total knee replacement; PPV = positive predictive value; $\mathrm{NPV}=$ negative predictive value; $\mathrm{LR}^{+}=$positive likelihood ratio; $\mathrm{LR}-=$ negative likelihood ratio; $\mathrm{DOR}=$ diagnostic odds ratio.

${ }^{*}$ Cut-off values are presented in numbers of cells $/ \mu \mathrm{L}$.

\section{Diagnostic utility of SWCC}

The ROC plots to determine an optimal cut-off value for the SWCC had the area under curve of $0.974(95 \% \mathrm{CI}$; $0.945-1.000$ ) for all groups together (regardless of the site). According to the Youden index J method, the cut-off value for the SWCC with an optimal balance of the true positive rate (sensitivity) and false positive rate (1-specificity) was 3,450 cells $/ \mu \mathrm{L}$ (Fig. 2a). The cut-off values for the SWCC were 4,100 cells $/ \mu \mathrm{L}$ and 3,200 cells $/ \mu \mathrm{L}$ (positive $=$ values greater than or equal to them) when analyzed separately for THRs and TKRs, respectively. Corresponding diagnostic characteristics for each group in the study are shown in Table 3.

As a result, the LR+ for all the SWCC cut-offs was high enough to influence significantly the diagnosis of PJI. Assuming a patient has a pre-test probability of PJI $50 \%$, the post-test probability of PJI is $95 \%$ when the test is positive, and $5.6 \%$ when the test is negative (valid for $\mathrm{LR}+=19.0$ and $\mathrm{LR}_{-}=0.06$ ).

\section{Diagnostic utility of neutrophil and lymphocyte percentages}

The ROC plots to determine an optimal cut-off value for the neutrophil and lymphocyte percentages had the area un- der curve of 0.962 (95\% CI; $0.939-0.984)$ and 0.951 (95\% CI; 0.924 - 0.978) for all the groups together, respectively (Fig. 2b, c). The cut-off values for neutrophil and lymphocyte percentages were $74.6 \%$ (positive $=$ values greater than or equal to them) and $14.6 \%$ (valid for values less than or equal to them), respectively, for all the groups under study. The optimal cut-off values for neutrophil percentages were $76.5 \%$ and $72.8 \%$ when analyzed separately for THRs and TKRs. The data for lymphocyte percentages were $14.6 \%$ and $19.9 \%$ for THRs and TKRs, respectively. Corresponding diagnostic characteristics are shown in Table 3.

Like the case of the SWCC, the LR for neutrophil percentages has the ability to change significantly the pre-test probability of PJI. Again, let's assume that the patient we are caring for has a pre-test probability of PJI 70\% (based on history, physical examination, and elevated CRP or IL-6). The change of the pre-test probability as a result of neutrophil or lymphocyte percentage is shown in Fig. 3.

\section{Combination of the SWCC}

\section{with neutrophil/lymphocyte percentage}

Since we had all the data available for the majority of patients from whom joint fluid samples were taken preoperatively, it was necessary to interpret them collectively. 


\section{Model for quantitative data}

We wanted to know whether a joint with a particular value of the SWCC, neutrophil and lymphocyte percentages should be considered as infected or aseptic. The probability that a patient has an infection can be calculated using the binomic logistic regression model (characteristics of this model: -2 log likelihood = 56.938; Cox $\&$ Schnell R square $=0.584$; and Nagelkerke $\mathrm{R}$ square $=$ 0.898):

$p r o b=\frac{e^{(-5.383+0.299 * \text { Leu }+0.043 * \text { Neu }-0.049 * \text { Lymfo })}}{\left(1+e^{(-5.383+0.299 * \text { Leu }+0.043 * \text { Neu }-0.049 * \text { Lymfo })}\right)}$

If prob is higher than 0.5 , then the data are in favor of infection. If prob is lower than 0.5 , then the data are against infection. Let's consider an example with the SWCC $=22,000$ cells $/ \mu \mathrm{L}$, neutrophil percentage $=33 \%$ and lymphocyte percentage $=67 \%$ :

$$
\begin{gathered}
\text { prob }=\frac{e^{(-5.383+0.299 * 22+0.043 * 33-0.049 * 67)}}{\left(1+e^{(-5.383+0.299 * 22+0.043 * 33-0.049 * 67)}\right)}= \\
=\frac{e^{(-0.669)}}{\left(1+e^{(-0.669)}\right)}=0.3387
\end{gathered}
$$

As prob is lower than 0.5 , this patient should be considered as non-infected despite the fact that the SWCC is much higher than the relevant cut-off point (i.e. 3,450 cells $/ \mu \mathrm{L})$.

The same model can be applied separately on THA and TKA patients with the following characteristics: $-2 \mathrm{log}$ likelihood 23.025 and 32.704; Cox \& Schnell R square 0.505 and 0.629 ; and Nagelkerke $\mathrm{R}$ square 0.878 and 0.910 , respectively.

The probability of THA infection can be calculated using the formula:

$$
\text { prob }=\frac{e^{(-2.665+0.269 * \text { Leu }+0.013 * \text { Neu }-0.091 * \text { Lymfo })}}{\left(1+e^{(-2.665+0.269 * \text { Leu }+0.013 * \text { Neu }-0.091 * \text { Lymfo })}\right)}
$$

The probability of TKA infection can be calculated using the formula:

$$
\text { prob }=\frac{e^{(-6.958+0.299 * \text { Leu }+0.063 * \text { Neu }-0.025 * \text { Lymfo })}}{\left(1+e^{(-6.958+0.299 * \text { Leu }+0.063 * \text { Neu }-0.025 * \text { Lymfo })}\right)}
$$

Let's consider that a patient with THR has the SWCC $=5,130$ cells $/ \mu \mathrm{L}$, neutrophil percentage $=70.6 \%$ and $1 \mathrm{ym}-$ phocyte percentage $=28.2 \%$ :

$$
\begin{gathered}
\text { prob }=\frac{e^{(-2.665+0.269 * 5.13+0.013 * 70.6-0.091 * 28.2)}}{\left(1+e^{(-2.665+0.269 * 5.13+0.013 * 70.6-0.091 * 28.2)}\right)}= \\
=\frac{e^{(-2.933)}}{1+e^{(-2.933)}}=0.0505
\end{gathered}
$$

Let's assume the same case as above but under assumption it is a patient with TKR:

$$
\begin{gathered}
\text { prob }=\frac{e^{(-6.958+0.299 * 5.13+0.063 * 70.6-0.025 * 28.2)}}{\left(1+e^{(-6.958+0.299 * 5.13+0.063 * 70.6-0.025 * 28.2)}\right)}= \\
=\frac{e^{(-1.6813)}}{1+e^{(-1.6813)}}=0.1569
\end{gathered}
$$

As both examples result in prob $<0.5$, the outcomes are against the diagnosis of PJI.

\section{Model for qualitative data}

The second model was developed to calculate with categories $(0=$ negative outcome, and $1=$ positive outcome) instead of distinct figures. When the model was applied for 1 defined as more than 3,450 cells $/ \mu \mathrm{L}$ and $74.6 \%$ of neutrophil percentage, and lower than $14.6 \%$ of lymphocyte percentage, the parameters of the model were as follows: $-2 \log$ likelihood $=89.413$; Cox \& Schnell R square $=0.541$; and Nagelkerke R square $=0.832$.

Probability that a patient with binary outcomes of the SWCC, neutrophil percentage, and lymphocyte percentage has an infection can be calculated using the formula:

$$
\text { prob }=\frac{e^{(-5.127+3.924 * \text { Leu }+1.683 * \text { Neu }+1.880 * \text { Lymfo })}}{\left(1+e^{(-5.127+3.924 * \text { Leu }+1.683 * \text { Neu }+1.880 * \text { Lymfo })}\right)}
$$

Using the same example as above and transcription with regard to infection with the positive SWCC $(22,000$ $>3,450$ cells $/ \mu \mathrm{L})$ but negative neutrophil percentage $(33 \%$ $<74.6 \%)$ and negative lymphocyte percentage $(67 \%>$ $14.6 \%)$, the model results in prob $<0.5$, hence against the infection:

$$
\begin{gathered}
\text { prob }=\frac{e^{(-5.127+3.924 * 1+1.683 * 0+1.880 * 0)}}{\left(1+e^{(-5.127+3.924 * 1+1.683 * 0+1.880 * 0)}\right)}=: \\
=\frac{e^{(-1.2030)}}{1+e^{(-1.2030)}}=0.2309
\end{gathered}
$$

The same model can be applied to the cut-off points for THA ( 1 is for outcomes higher than 4,100 cells $/ \mu \mathrm{L}$, $76.5 \%$ of neutrophil percentage, and lower than $14.6 \%$ of lymphocyte percentage) and TKA ( 1 is for outcomes higher than 3,200 cells $/ \mu \mathrm{L}, 72.8 \%$ of neutrophil percentage, and lower than $19.90 \%$ of lymphocyte percentage) locations (-2 log likelihood 23.128 and 51.205; Cox \& Schnell R square 0.505 and 0.588 ; and Nagelkerke R square 0.877 and 0.852 , respectively).

The estimate of probability of infection in patients with THR is:

$$
\text { prob }=\frac{e^{(-6.330+4.583 * \text { Leu }+4.786 * \text { Neu }+0.196 * \text { Lymfo })}}{\left(1+e^{(-6.330+4.583 * \text { Leu }+4.786 * \text { Neu }+0.196 * \text { Lymfo })}\right)}
$$

The estimate of probability of infection in patients with TKR is:

prob $=\frac{e^{(-4.718+4.643 * \text { Leu }+2.036 * \text { Neu }+0.480 * \text { Lymfo })}}{\left(1+e^{(-4.718+4.643 * \text { Leu }+2.036 * \text { Neu }+0.480 * \text { Lymfo })}\right)}$ 
Table 4. Showing the cut-off points for PJI diagnosing in several preceding studies.

\begin{tabular}{|c|c|c|c|c|c|c|c|}
\hline \multirow[t]{2}{*}{ Author } & \multirow[t]{2}{*}{ Location } & \multicolumn{3}{|c|}{ SWCC (cells $/ \mu \mathrm{L})$} & \multicolumn{3}{|c|}{ Neutrophils (\%) } \\
\hline & & Cut-off & $\mathrm{Se}$ & $\mathrm{Sp}$ & Cut-off & $\mathrm{Se}$ & $\mathrm{Sp}$ \\
\hline Parvizi, Gehrke, 2014 & Hip or Knee & $3,000 / \mu \mathrm{L}$ & $\mathrm{N} / \mathrm{A}$ & N/A & $80 \%$ & $\mathrm{~N} / \mathrm{A}$ & $\mathrm{N} / \mathrm{A}$ \\
\hline \multirow[t]{3}{*}{ Dinneen et al, 2013} & Hip or Knee & $1,590 / \mu \mathrm{L}$ & 0.895 & 0.913 & $65 \%$ & 0.897 & 0.866 \\
\hline & Hip & $1,425 / \mu \mathrm{L}$ & $\mathrm{N} / \mathrm{A}$ & $\mathrm{N} / \mathrm{A}$ & $65 \%$ & $\mathrm{~N} / \mathrm{A}$ & $\mathrm{N} / \mathrm{A}$ \\
\hline & Knee & $1,715 / \mu \mathrm{L}$ & $\mathrm{N} / \mathrm{A}$ & $\mathrm{N} / \mathrm{A}$ & $54 \%$ & N/A & $\mathrm{N} / \mathrm{A}$ \\
\hline Cipriano et al, 2012 & Hip or Knee & $3,450 / \mu \mathrm{L}$ & 0.91 & 0.93 & $78 \%$ & 0.955 & 0.873 \\
\hline Zmistowski et al, 2012 & Knee & $3,000 / \mu \mathrm{L}$ & 0.93 & 0.94 & $75 \%$ & 0.93 & 0.83 \\
\hline Shukla et al, 2010 & Hip & $3,528 / \mu \mathrm{L}$ & 0.78 & 0.96 & $79 \%$ & 0.78 & 0.82 \\
\hline Lee et al, 2010 & Knee & $3,800 / \mu \mathrm{L}$ & 0.86 & 0.79 & $89 \%$ & 0.72 & 0.62 \\
\hline Schinski et al, 2008 & Hip & $4,200 / \mu \mathrm{L}$ & 0.84 & 0.94 & $80 \%$ & 0.84 & 0.82 \\
\hline Ghanem et al, 2008 & Knee & $1,100 / \mu \mathrm{L}$ & 0.907 & 0.881 & $64 \%$ & 0.95 & 0.947 \\
\hline Della Valle et al, 2007 & Knee & $3,000 / \mu \mathrm{L}$ & 1.00 & 0.98 & $65 \%$ & 0.976 & 0.849 \\
\hline Parvizi et al, 2006 & Hip or Knee & $1,760 / \mu \mathrm{L}$ & 0.90 & 0.99 & $73 \%$ & 0.93 & 0.95 \\
\hline Trampuz et al, 2004 & Knee & $1,700 / \mu \mathrm{L}$ & 0.94 & 0.88 & $65 \%$ & 0.97 & 0.98 \\
\hline Mason et al, 2003 & Knee & $2,500 / \mu \mathrm{L}$ & 0.69 & 0.98 & $60 \%$ & 0.75 & 0.90 \\
\hline Spangehl et al, 1999 & Hip & $50,000 / \mu \mathrm{L}$ & 0.36 & 0.99 & $80 \%$ & 0.89 & 0.85 \\
\hline \multirow[t]{3}{*}{ Current study } & Hip or Knee & $3,450 / \mu \mathrm{L}$ & 0.947 & 0.950 & $74.6 \%$ & 0.931 & 0.991 \\
\hline & Hip & $4,100 / \mu \mathrm{L}$ & 0.920 & 0.968 & $76.5 \%$ & 0.957 & 0.938 \\
\hline & Knee & $3,200 / \mu \mathrm{L}$ & 0.960 & 0.959 & $72.8 \%$ & 0.939 & 0.900 \\
\hline
\end{tabular}

SWCC = synovial white cell count; $\mathrm{Se}=$ sensitivity, $\mathrm{Sp}=$ specificity; $\mathrm{PJI}=$ prosthetic joint infection.

\section{DISCUSSION}

In this study, we found that the SWCC and differential count of leukocytes analysis of JFS showed very good diagnostic characteristics for changing significantly the pre-test probability of PJI. Our results identified the cutoff value for the SWCC of 3,450 cells $/ \mu \mathrm{L}$ and for neutrophil percentage of $74.6 \%$. When the anatomical location was included, then the same figures were 4,100 cells $/ \mu \mathrm{L}$ and $76.5 \%$ for THR and 3,200 cells/ $\mu \mathrm{L}$ and $72.8 \%$ for TKR, respectively. In addition, very impressive diagnostic characteristics were revealed for the lymphocyte joint fluid count. Finally, we introduced the model enabling simultaneous interpretation of the SWCC, neutrophil and lymphocyte percentages.

Compared to previous studies, the optimal SWCC cutoff points in our study are higher, especially at the site of the knee (Table 4). The reason for the difference may lie at least partly in the definition of PJI used in particular studies, patients included, methodology and/or organizational differences among studies. Our study excluded patients with chronic inflammatory diseases like crystal arthropathies (e. g. gout, pseudogout) and tuberculosis. However, we included patients with rheumatoid arthritis contrary to other authors ${ }^{14-16}$ who excluded all patients with chronic inflammatory joint diseases despite the fact that at least one study showed that the SWCC and differential count of leukocytes have similar cut-off values and overall testing performance in patients with chronic inflammatory arthritis compared to those with non-in- flammatory arthritis ${ }^{17}$. Others, for example Schinsky ${ }^{18}$, do not report such exclusion. Some studies excluded patients with antibiotic pretreatment ${ }^{15,18}$. In contrast, $47 \%$ of the infected patients in our study were treated with antibiotics prior to their operation. Dinneen et al. also excluded patients who were within six weeks of their primary TKR or THR as the post-operative inflammatory state would be likely to affect the normal SWCC and proportion of neutrophils in the aspirate ${ }^{14}$. Such patients were admitted to our study and were classified as early postoperative infection. At least one study reported optimal cut-off values of the SWCC and neutrophils for the diagnosis of PJI in the early postoperative period supporting their position in the diagnostic workout ${ }^{19}$. In order to eliminate the role of physicians participating in the care, one of the team members who had participated neither in the diagnostic process nor in the treatment process reclassified all the treated patients as infected or controls several months after revision surgeries when all the data related to PJI were available. A design-related bias is a potential source of inter-study discrepancies ${ }^{20}$. Here we conducted a prospective study of a very simple design; a well-known index test was evaluated comparing the results of JFS cytology with an independent reference standard, and appropriate statistics were employed. On the other hand, not all the requirements contained in STARD 2015 (ref. ${ }^{21}$ ) were addressed in this study, partly due to the fact that the protocol of the study was planned between 2006 and 2008, and the permission of the Ethical Committee was obtained in 2009. 
The value of a diagnostic test depends on how much the test changes the pre-test probability of a disease. PJI is defined as occurrence of vital and replicating bacteria on an implant surface and/or in the periprosthetic environment. Hence, there is no doubt that the finding of positive cultures makes the diagnosis. However, the contact of bacteria with host cells induces a complex response that is not exclusive for infection. This means for instance that neutrophils can be present in joint fluid even if there are no bacteria in the artificial joint. Under such circumstances, the quantity of leucocytes and an appropriate amount of their cellular fractions can be associated with prosthetic joint infection. A recent meta-analysis found excellent diagnostic power of the SWCC and neutrophil percentage $^{7}$. The AUC was for the SWCC (0.96) and for neutrophil percentage $(0.95)$ as well as the pooled sensitivity (0.88) and specificity (0.93) for PJI detection for both the SWCC and neutrophil percentage are fully comparable with our study. To demonstrate the diagnostic contribution of the SWCC and neutrophil percentage to PJI diagnosis/exclusion, the authors of the meta-analysis used the likelihood ratio scattergram. The starting point of $20 \%$ probability of PJI is relatively low because we do not know what this means in clinical terms. On the other hand, their example shows very well the diagnostic power of both the SWCC and neutrophil percentage. In our example, we used an increased probability of infection (70\%) because we believe that the test (i.e. puncture of a joint followed by cytology, microbiology, other examinations) should be indicated only in patients showing probability of 50\% and higher (i.e. when PJI is more probable than a chance). In addition, our study revealed potentially useful characteristics also for lymphocyte percentage.

Looking at the literature, all the studies reported the results of the SWCC and neutrophil percentage as independent while these are together with lymphocyte percentage highly dependent on each other in the description of the biological status of a joint. Moreover, all these variables are reported jointly by a laboratory. Finally, there are some patients who have increased SWCC while the neutrophil percentage is low and lymphocyte percentage is high. Therefore, there is a need to interpret these figures simultaneously in order to increase accuracy of cytological examination of JFS (ref. ${ }^{22}$ ). However, in the bible of evidence based clinical approach, Furukawa et al. write: "If 2 tests are very closely related, however, the application of the second test (here neutrophil and/or lymphocyte percentages) may provide little or no additional information, and the sequential application of LRs will yield misleading results" ( ref. $^{23}$ ). Therefore, we used the logistic binomic regression models either to include binary or particular values of the SWCC, neutrophil and lymphocyte percentages into the estimation of probability of PJI (i.e. probability that an observation falls into the diseased/non-diseased category based on imported outcomes). Logistic regression can be used to construct decision rules, reflecting the combined diagnostic value of a number of diagnostic variables ${ }^{24,25}$.

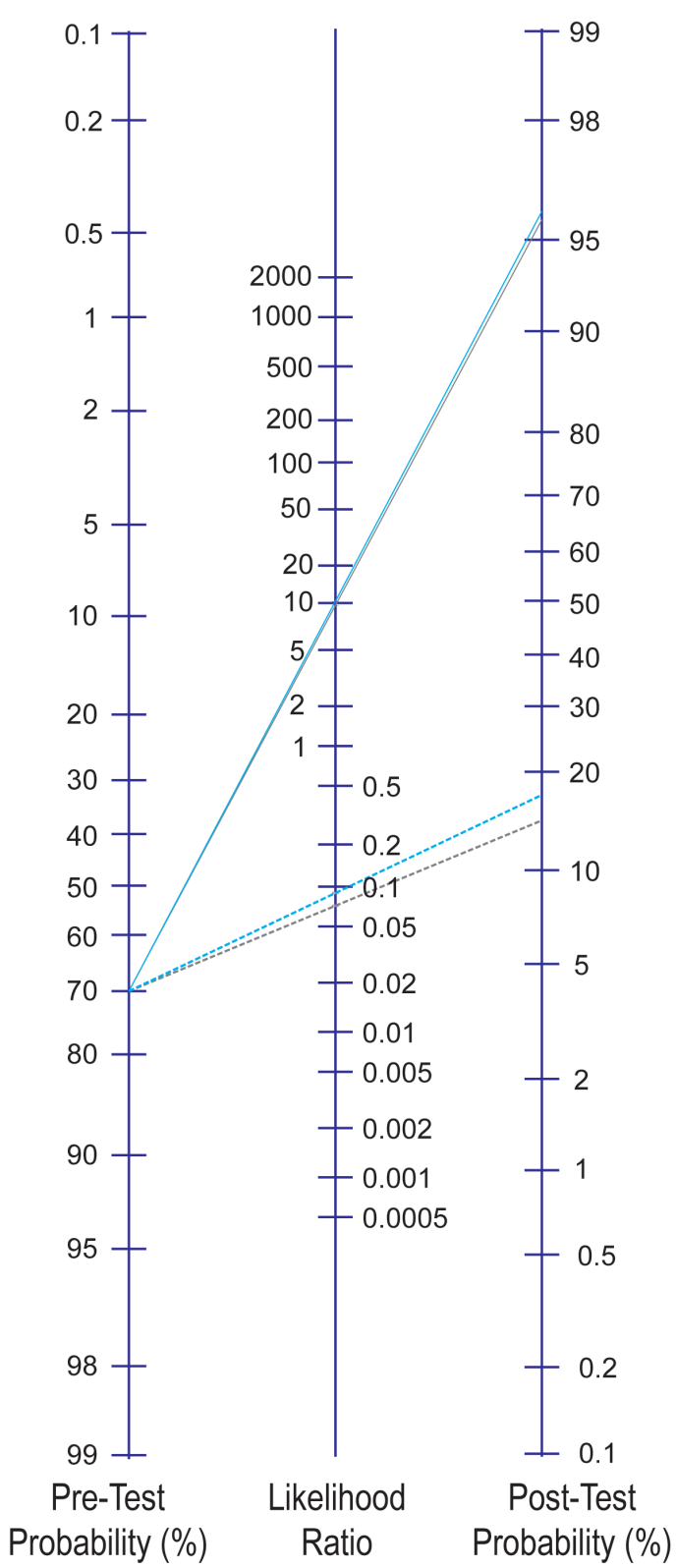

Fig. 3. Estimation of the post-test probability of prosthetic joint infection for the neutrophil or lymphocyte percentages when the pre-test probability is $70 \%$; the blue lines are for the neutrophil percentage; the green lines are for the lymphocyte percentage; the dashed lines are for negative results.

\section{CONCLUSION}

In summary, this study strongly supports the usage of the SWCC and neutrophil percentage in the diagnostic workout for PJI. In addition, the role for the lymphocyte count is emphasized based on its diagnostic characteristics. On the other hand, the optimal strategy for integrating data from all cytological methods remains to be proved. Here we described the logistic regression model. Theoretically, other bivariate models or the hierarchical models as described in the Cochrane handbook can be used. Regardless of the chosen approach a large prospective study will be necessary to validate the multi-component probabilistic approach to PJI diagnostics. 


\section{ABBREVIATIONS}

AUC, area under the curve; CI, confidence interval; CRP, C-reactive protein; DOR, diagnostic odds ratio; EDTA, ethylenediaminetetraacetic acid; ESR, erythrocyte sedimentation rate; HPF, high power field; IL-6, interleukin 6; JFS, joint fluid sample; LR, likelihood ratio; MSIS, Musculoskeletal Infection Society; NPV, negative predictive value; PJI, prosthetic joint infection; PMN, polymorphonuclear; PPV, positive predictive value; ROC, receiver operating characteristic; Se, sensitivity; SF, synovial fluid; Sp, specificity; SWCC, synovial white cell count; THR, total hip replacement; TKR, total knee replacement.

Acknowledgements: Funding was obtained from the Ministry of Health of the Czech Republic (AZV project No. 15-27726A).

The authors thank Yvona Loveckova, MD, PhD, Department of Microbiology, Faculty of Medicine and Dentistry, Palacký University Olomouc, University Hospital Olomouc, Czech Republic for collaboration in the microbiological part of the study, and Jiri Ehrmann, MD, PhD, prof. and Tomas Tichy, MD, Department of Pathology, Faculty of Medicine and Dentistry, Palacký University Olomouc, University Hospital Olomouc, Czech Republic for histopathological analyses performed as part of the study.

Author contributions: JG gave the ideas, designed the manuscript, and wrote the manuscript. JJ guaranteed the cytological data and contributed to the writing of the parts of the manuscript related to joint fluid cytology. MS collected all the data, and therefore guaranteed them. $\mathrm{He}$ also contributed to the writing of the manuscript. JZ performed the entire statistical analysis including the logistic binomic regression for simultaneous evaluation of joint fluid cytology. All the authors read and approved the final manuscript.

Ethics and consent to participate: Ethical approvals for this study have been obtained from the Ethical Committee of the University Hospital Olomouc and the Faculty of Medicine and Dentistry, Palacký University Olomouc on 1 September 2008 and 16 December 2009.

Conflict of interest statement: The authors state that there are no conflicts of interest regarding the publication of the article.

Availability of data and materials: All relevant raw data will be freely available to any scientist wishing to use them for non-commercial purposes, without breaching participant confidentiality. If interested, please contact the corresponding author.

\section{REFERENCES}

1. Parvizi J, Gehrke T, International Consensus Group on Periprosthetic Joint I. Definition of periprosthetic joint infection. J Arthroplasty 2014;29(7):1331.

2. Esteban J, Sorli L, Alentorn-Geli E, Puig L, Horcajada JP. Conventional and molecular diagnostic strategies for prosthetic joint infections. Expert Rev Mol Diagn 2014;14(1):83-96.
3. Piper KE, Fernandez-Sampedro M, Steckelberg KE, Mandrekar JN, Karau MJ, Steckelberg JM, Berbari EF, Osmon DR, Hanssen AD, Lewallen DG, Cofield RH, Sperling JW, Snachez-Sotelo J, Huddleston PM, Dekutoski MB, Yaszemski M, Currier B, Patel R. C-reactive protein, erythrocyte sedimentation rate and orthopedic implant infection. PLoS One 2010;5(2):e9358.

4. Parvizi J, Alijanipour P, Barberi EF, Hickok NJ, Phillips KS, Shapiro IM, Schwarz EM, Stevens MH, Wang Y, Shirtliff ME. Novel developments in the prevention, diagnosis, and treatment of periprosthetic joint infections. J Am Acad Orthop Surg 2015;23 Suppl:S32-43.

5. Parvizi J, Della Valle CJ. AAOS Clinical Practice Guideline: diagnosis and treatment of periprosthetic joint infections of the hip and knee. J Am Acad Orthop Surg 2010;18(12):771-2.

6. Borens $\mathrm{O}$, Corvec S, Trampuz A. Diagnosis of periprosthetic joint infections. Hip Int 2012;22 Suppl 8:S9-14.

7. Qu X, Zhai Z, Liu X, Li H, Wu C, Li Y, Li H, Zhu Z, Qin A, Dai K. Evaluation of white cell count and differential in synovial fluid for diagnosing infections after total hip or knee arthroplasty. PLoS One 2014;9(1):e84751.

8. Gallo J, Kolar M, Dendis M, Loveckova Y, Sauer P, Zapletalova J, Koukalova D. Culture and PCR analysis of joint fluid in the diagnosis of prosthetic joint infection. New Microbiol 2008;31(1):97-104.

9. Trampuz A, Zimmerli W. Prosthetic joint infections: update in diagnosis and treatment. Swiss Med Wkly 2005;135(17-18):243-51.

10. Holinka J, Bauer L, Hirschl AM, Graninger W, Windhager R, Presterl E. Sonication cultures of explanted components as an add-on test to routinely conducted microbiological diagnostics improve pathogen detection. J Orthop Res 2011;29(4):617-22.

11. Atkins BL, Athanasou N, Deeks JJ, Crook DW, Simpson H, Peto TE, McLardy-Smith P, Berendt AR. Prospective evaluation of criteria for microbiological diagnosis of prosthetic-joint infection at revision arthroplasty. The OSIRIS Collaborative Study Group. J Clin Microbiol 1998;36(10):2932-9.

12. Mirra JM, Amstutz HC, Matos M, Gold R. The pathology of the joint tissues and its clinical relevance in prosthesis failure. Clin Orthop Relat Res 1976(117):221-40.

13. Luo J, Xiong C. Youden index and Associated Cut-points for Three Ordinal Diagnostic Groups. Commun Stat Simul Comput 2013;42(6):1213-1234.

14. Dinneen A, Guyot A, Clements J, Bradley N. Synovial fluid white cell and differential count in the diagnosis or exclusion of prosthetic joint infection. Bone Joint J 2013;95-B(4):554-7.

15. Spangehl MJ, Masri BA, O'Connell JX, Duncan CP. Prospective analysis of preoperative and intraoperative investigations for the diagnosis of infection at the sites of two hundred and two revision total hip arthroplasties. J Bone Joint Surg Am 1999;81(5):672-83.

16. Ghanem E, Parvizi J, Burnett RS, Sharkey PF, Keshavarzi N, Aggarwal A, Barrack RL. Cell count and differential of aspirated fluid in the diagnosis of infection at the site of total knee arthroplasty. J Bone Joint Surg Am 2008;90(8):1637-43.

17. Cipriano CA, Brown NM, Michael AM, Moric M, Sporer SM, Della Valle CJ. Serum and synovial fluid analysis for diagnosing chronic periprosthetic infection in patients with inflammatory arthritis. J Bone Joint Surg Am 2012;94(7):594-600.

18. Schinsky MF, Della Valle CJ, Sporer SM, Paprosky WG. Perioperative testing for joint infection in patients undergoing revision total hip arthroplasty. J Bone Joint Surg Am 2008;90(9):1869-75.

19. Yi PH, Cross MB, Moric M, Sporer SM, Berger RA, Della Valle CJ. The 2013 Frank Stinchfield Award: Diagnosis of infection in the early postoperative period after total hip arthroplasty. Clin Orthop Relat Res 2014;472(2):424-9.

20. Lijmer JG, Mol BW, Heisterkamp S, Bonsel GJ, Prins $\mathrm{MH}$, van der Meulen JH, Bossuyt PM. Empirical evidence of design-related bias in studies of diagnostic tests. JAMA 1999;282(11):1061-6.

21. Bossuyt PM, Reitsma JB, Bruns DE, Gatsonis CA, Glasziou PP, Irwig L, Lijmer JG, Moher D, Rennie D, de Vet HC, Kressel HY, Rifai N, Golub RM, Altman DG, Hooft L, Korevaar DA, Cohen JF, for the STARD group. STARD 2015: an updated list of essential items for reporting diagnostic accuracy studies. BMJ 2015;351:h5527.

22. Pepe MS, Thompson ML. Combining diagnostic test results to increase accuracy. Biostatistics 2000;1(2):123-40.

23. Furukawa TA, Strauss, S.E., Bucher, H.C., Agoritsas, T., Guyatt, G. Diagnostic tests. Guyatt G, editor. New York: McGraw-Hill Professional; 2014. 345-357 p. 
24. Glas AS, Lijmer JG, Prins MH, Bonsel GJ, Bossuyt PM. The diagnostic odds ratio: a single indicator of test performance. J Clin Epidemiol 2003;56(11):1129-35.

25. Li J, Fine JP. ROC analysis with multiple classes and multiple tests: methodology and its application in microarray studies. Biostatistics 2008;9(3):566-76. 